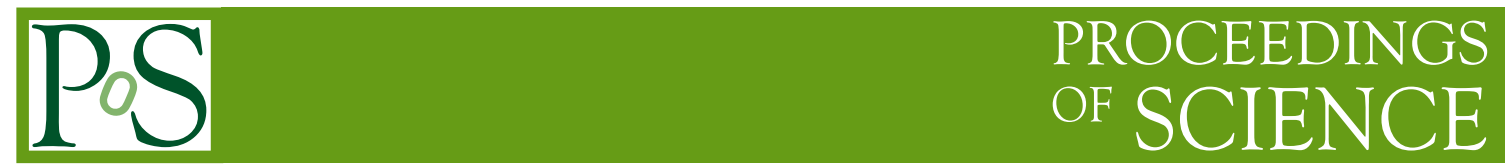

\title{
Astrophysical Fashions - A Warning
}

\section{Wolfgang Kundt*}

Argelander Institut of Bonn University, Germany

E-mail: wkundteastro.uni-bonn.de

Some 14 more or less tacit, but crucial assumptions will be highlighted which have been frequently made in standard literature on geophysical, astrophysical, and (even) biophysical problems - between 1978 and 2016 - and proposed to be replaced by alternative assumptions.

Frontier Research in Astrophysics - II

23-28 May 2016

Mondello (Palermo), Italy

${ }^{*}$ Speaker. 


\section{Astrophysical Alternatives}

(1) Distances: A fundamental and nontrivial problem in astrophysics is to measure the distances of observed objects, in particular when they are far from us; whereby "far" begins already near $10^{8} \mathrm{~cm}$ for certain terrestrial problems, e.g. in deserts, or on oceans, and gets increasingly more difficult to determine when the edge of the observable Universe is approached, at distances of $\lesssim 10^{28} \mathrm{~cm}$. On those largest scales, distances tend to be measured in units of cosmic recession velocities at trans-luminal (expansion) speeds, whereby spectral redshift $\mathrm{z}$ and Lorentz factor $\gamma$ are related by the well-known special-relativistic law:

$$
z+1=\gamma(1+\beta)
$$

and take the values $\{\mathrm{z}, \gamma\}=\{9,5\}$ in extreme cases for the well-known gamma-ray bursts (GRBs) (of which 3 on average strike our planet per day); $\mathrm{z}:=\mathrm{d} \mathrm{H} / \mathrm{c}, \mathrm{H}:=\dot{R} / R, \gamma:=1 / \sqrt{1-\beta^{2}}$. For this reason, GRBs tend to be judged emitted at extragalactic distances in the mainstream literature without any convincing, generally accepted mechanism and/or sources - rather than as centrifugal mass ejections by nearby disk-fed rotating neutron stars in our Galaxy, two vastly different interpretations already because of the vastly different involved energies of the (hard!) emissions.

Another, independent method of measuring distances of GRBs - and of similar bursting events is their association with distant galaxies called 'host galaxies', whose redshifts agree with those of the (few spectral lines in the) bursts. Such host galaxies are often no more than unresolved bright spots in the sky, with only one or very few spectral lines of the same redshift, and are more often absent than present. They can be (convincingly) re-interpreted (by me) as being emitted during the collisional interactions of ejected splinters with ambient matter, i.e. as being created by the burst, in its immediate neighbourhood, and even as being independently observed as its (broadband) afterglow. Having looked carefully at some of their best cases, I have arrived at the conclusion that so far, all of the (many!) published 'host galaxies' have been fake. Note that an identification of a distant point source as a "host galaxy" ought to include a feasibility estimate: whether or not a galaxy of that (large) redshift can be expected capable of emitting such super-energetic bursts of radiation.

A question often asked in this connection is why we do not simultaneously see blueshifts in the spectra, emitted by the approaching splinters. In order to answer this question, please look at figure 1 - called light echo geometry - which shows a 2-d section through a burst, its observer, and a few of the light rays emitted by the burst and reflected, or scattered by intervening nebulous matter. All rays leaving the left focal point of the drawn ellipsoid simultaneously (at speed c) reach the observer in its right focal point likewise simultaneously, i.e. would be visible in a (wide angle) snapshot. When the observer focusses on the burst, he or she would see its initial flash blueshifted, for a timespan of the duration of its flash, but would see all later emissions redshifted in the original viewing direction, coming from the receding side of the cloud of ejected burst fragments, (assumed spherical). Such (partially) blueshifted, early emissions, of duration $\lesssim s e c$, have not been reported yet for any GRB; they would be difficult to be detected; cf. [Kundt, 2010, 2014a], [Kundt \& Marggraf, 2014]. In my understanding, all GRBs reach us from inside our Milky Way. 


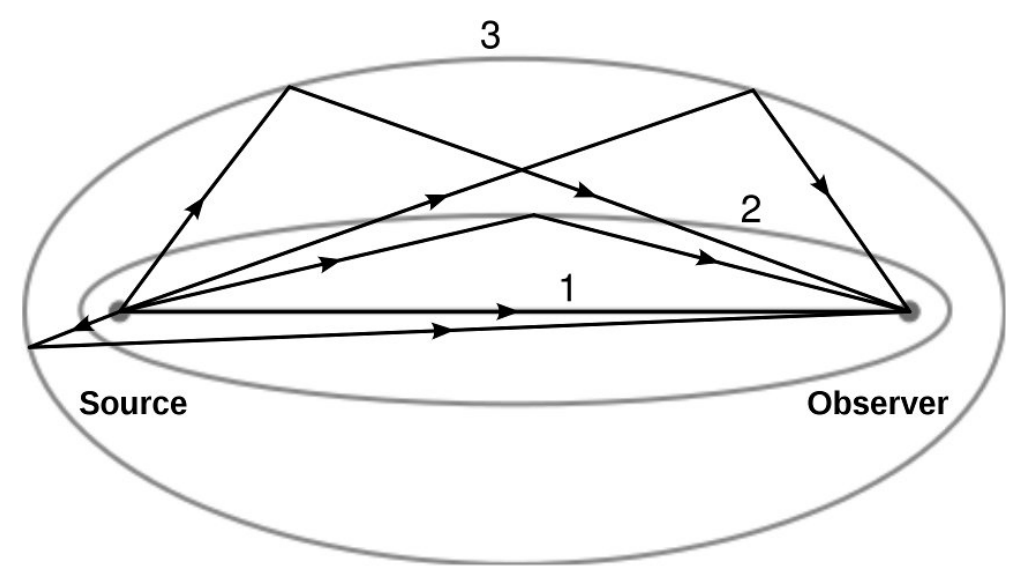

Figure 1: Spacetime Geometry of a Light Echo: All emissions leaving the Source Location simultaneously at the same speed reach the Observer Location likewise simultaneously, if scattered, reflected, or otherwise re-emitted somewhere along the ellipsoid drawn in projection. In this way, Galactic $\mathrm{SNe}$ from the past millennium have meanwhile been re-detected.

(2) Explosions: Not only on Earth, but also in our cosmic surroundings can we observe occasional explosions, of various strength. They tend to be of stellar scale, and tend to be called "Novae" if moderate, and "Supernovae" if much brighter, ("novae" because they show up as "new" stars in the sky). For a few decades, novae were thought to be formations, or flarings of white dwarfs, whereas supernovae were thought to be formations of neutron stars. In the first case, the explosion energy stems from matter falling into the deep potential well of a degenerate dwarf star, in the second case of a neutron star, (whose potential well is a thousand times deeper, because of a $10^{3}$ times smaller radius, at comparable mass). What geometries do such explosions possess?

Whereas terrestrial explosions tend to be fountain-like, with their fragments raining down back to the Earth's surface sooner or later, celestial explosions can have escape energy such that their fragments recede in all directions, thereby escaping from the gravity field of their source. Two possibilities must be distinguished: The explosion energy can be deposited either in the enclosing medium, in the form of a (strong, outgoing) shock wave, or can be deposited in a huge number of fragments of the former container, each of them escaping along their own ballistic trajectories, in the form of a 'Hubble' flow. One speaks of \{pressure, splinter (shrapnel)\} bombs, respectively, the latter having the larger ranges. Important for a correct theoretical description is the finding that in the case of stellar explosions - with their strong gravitational fields - these two types of bomb result respectively for $\{\mathrm{NR}, \mathrm{ER}\}$ pistons, $\mathrm{NR}:=$ non-relativistic, $\mathrm{ER}:=$ extremely-relativistic, "piston" standing for the pushing medium, because these two pistons cool during their expansions as $\left\{\mathrm{r}^{-2}, \mathrm{r}^{-1}\right\}$ respectively with increasing radius, whereby only ER pistons succeed in forming successful ejections - later seen as SN remnants - whilst only NR pistons form (global) shock waves, but recollapse. Neither of the two types of bomb succeeds in ultimately boosting their matter to ER energies. Cosmic Ray (=: CR) production requires fast rotating, heavy magnets, see (3); [Kundt, 2008].

Figure 2 is a map of the "outflow in Orion", which I interpret as one of the youngest observed SN 


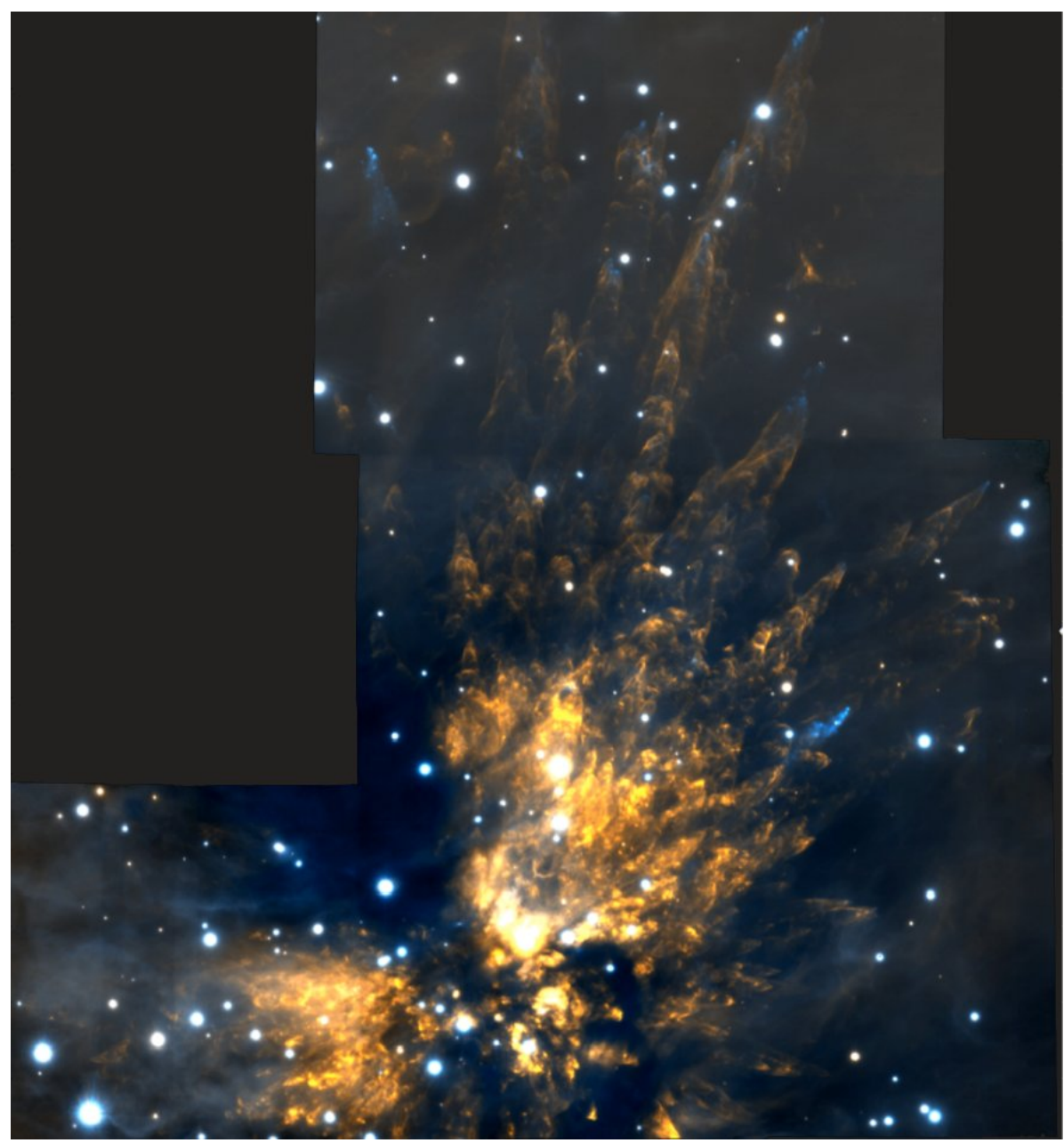

Figure 2: 'Outflow in Orion', interpreted by Aylin Yar and me [1997] as one of the youngest SN explosions in our Galaxy, (with Hubble-flow geometry).

remnants in our Galaxy, already because of its ordered - and energetic - Hubble-flow geometry, whilst distinguished colleagues of mine prefer to talk of "stellar outflows"; [Kundt \& Yar, 1997]. Explosions of heavy objects realise preferred geometries.

(3) Multistep Boosts, or 'shockwave accelerations', are often invoked in the astrophysical literature, in order to explain the origin of hard particles - or CRs - in an observed region, detected via their hard radiation. But multistep accelerations require perfect phasing, achieved in manmade accelerators (like at CERN), and in idealised calculations. Realistic cosmic setups, however, obey the second law of thermodynamics - the entropy law - according to which the conversion of random motions into ordered motions has a limited, tiny efficiency. Only in the test-particle limit does this efficiency reach useful, finite values, not applicable to the shock waves of the real world. As already anticipated in the last section, CR production wants heavy, fast rotators; [Kundt, 2009], [Kundt \& Marggraf, 2014]. Who is right?

(4) Conservation of 4-Momentum: is a strict relativistic law of whose validity we are convinced, 
but whose satisfaction is not always checked in cosmic applications, for instance in stellar-wind productions (of nearby stars), or of the precise size, and shape of our heliosphere, which separates the solar system from other neighbouring stellar systems. It therefore came as a surprise, between 2007 and 2011, that the two Voyager spacecrafts had not yet reached the outer edge of the heliosphere, (expected by me near $r=1$ lyr, hence reachable only within $\gtrsim 10^{4}$ years). And stellar-evolution theory quite often considers "photon-pressure driven winds", despite the extreme weakness of a photon's 3-momentum. Only indirectly, by heating immersed dusty components, can photons post-accelerate stellar windzones; [Kundt, 2012]. How many more decades will consensuses take to form, in these two trans-solar-system queries?

(5) Jet formation: How can unanimated celestial bodies blow jets, without a building plan like the DNA, or without a helping brain? Jets are observed to be blown by stars of various masses and ages, and even by the central engines of active galaxies, on scales between $10^{2} \mathrm{AU}$ and several $\mathrm{Mpc}$, with quite similar properties: hot cores, opening angles of order $1 \%$, bulk Lorentz factors $\gtrsim 10^{2}$, almost never branching, and with almost lossfree propagation. What medium propagates in them? For focussing and stability reasons alone (against environmental perturbations), I have expected the lightest existing substance as their (universal) constituing medium from the very beginning: $\mathrm{e}^{ \pm}$pair plasma, abundantly available from magnetic reconnections, buoyantly rising in deep gravitational potential wells, and easily to be channelled by toroidal, comoving magnetic fields, properties, all of which are available near heavy, magnetised rotators. Note that (dominantly) hadronic jet medii are ruled out by these criteria - for weight reasons alone - as well as black-hole engines of any size, independently of our recent insight that they do not form within general relativistic scenarios anyway, [Kundt, 1996, 2014b, 2015a].

These considerations rule out many proposed jet models, but are in line with [Kundt \& Krishna, 2004], [Kundt, 2015b], and with the well-studied jets in the nearby cD galaxy M 87 shown in figure 3. In particular, as Gopal Krishna and I have presented complete analytic solution classes (for both the moving charges, and their comoving electromagnetic fields, approximated cylindrically, instead of conically), numerical approximations will have a hard time when trying to find similarly intuitive classes of solutions. Jet formations probably rank highest in the set of all inorganic machines.

(6) Strengths of Magnetospheres: Neutron stars are now known to exist since 49 years, but none of them is near enough to Earth for having been mapped. In particular, we do not know the structure of their magnetospheres. How strong can their surface magnetic fields be, in favourable cases? Can they exceed $10^{14} \mathrm{G}$, as has been assumed by many scientists, in order to explain the so-called magnetars? I am not convinced, already for the simple reason that magnetic fields decay unless they are anchored in sufficiently dense (conductive) materials. Moreover, their unsteady spindown behaviour - with discrete spindowns and anti-glitches instead of discrete spinups and field decays, and with sporadic radiation epochs - suggests that they are all aged pulsars with collapsed vacuoles, embraced and confined by low-mass accretion disks (collected from their former windzones), which indent and enhance their frozen-in surface fields, and enhance their magnetic torques by factors of $\lesssim 10^{3}$. They may well be the (long-searched) dominant CR generators, and GRBers; cf. figure 4. Magnetospheric interactions are far from trivial.

(7) SN-like Lightcurves: As already discussed under (2), big stellar explosions radiate SN-like 


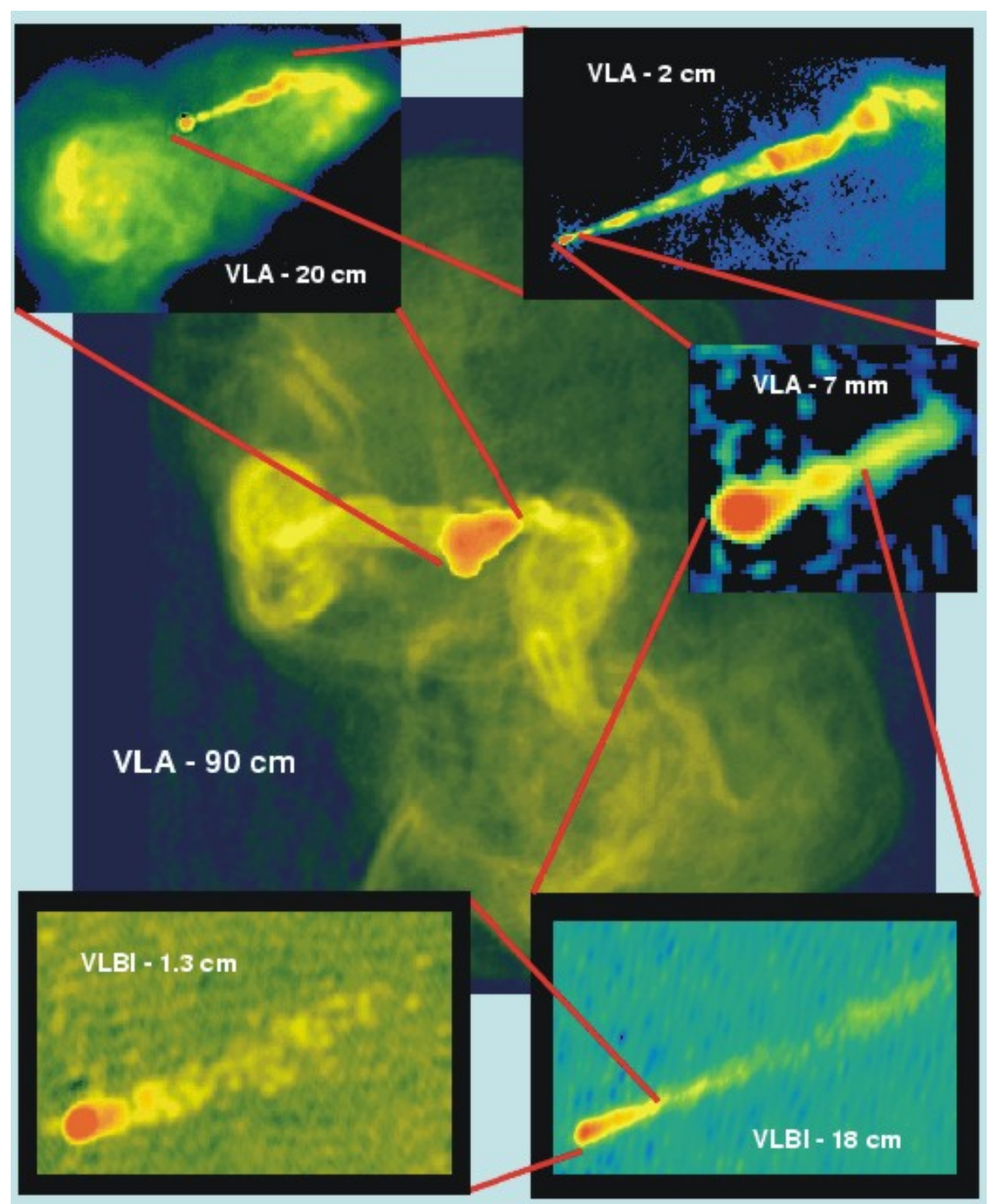

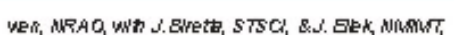

Figure 3: The nearest radio cD-galaxy - M87 - at distance $22 \mathrm{Mpc}$, of Eilek type B, shown at full size as well as at five successive enlargements, whose jets I interpret as blown by its central BD.

lightcurves, with their $\gtrsim$ four different temporal epochs, caused by the competition between a steadily growing luminous surface area and a steadily decreasing surface brightness, and with varying emission-line contributions from optically thin inclusions. Are these listed spectral properties also sufficient, to characterise explosions? I do not think so: A few GRBs have been recorded whose optical afterglows looked quite similar to SN lightcurves; they were thus interpreted as involving SN explosions. But what were their distances from us? And how large were the involved ejection speeds? Do GRBs share a relativistic component with SN explosions, via their centrifugal ejections? Astrophysics can involve diverse processes, with similar-looking phenomena, but with quite different underlying mechanisms; [Kundt, 2010]. In my understanding, the GRBs are 


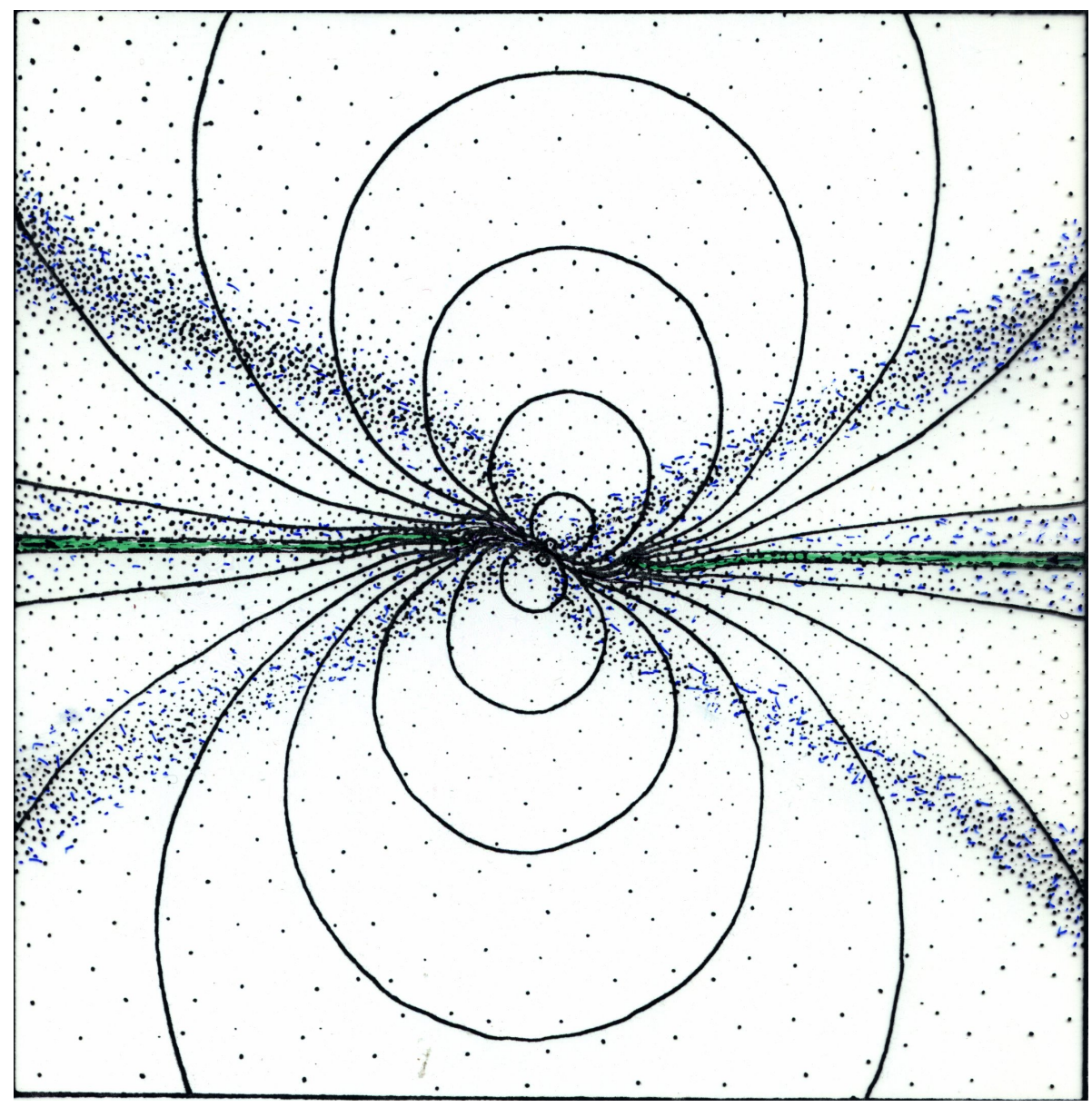

Figure 4: Exact shape of the (corotating) magnetosphere of a spinning neutron star, indented by a surrounding accretion disk (of somewhat distorted geometry near its inner edge); see [Horn \& Kundt, 1989], [Kundt, 1998a].

distinctly different from SN explosions.

(8) PSR Winds: how are they blown? How can a (heavy, compact) neutron star blow steadily matter off its surface, at relativistic speeds? Certainly not via gravitational forces, or even via thermal forces: the star's binding energy is marginally relativistic! Early offered solutions, by Peter Goldreich, Malvin Ruderman, Tom Gold, Curtis Michel, and by many others, involved electric forces, exerted by the corotating magnetosphere, via the Lorentz force $\mathbf{F}=\mathrm{e}(\mathbf{E}+\beta \times \mathbf{B})$, plus various pair formations, e.g. in transverse B-fields, by the Erber mechanism, cf. [Kundt, 1998a]. Even oscillatory discharges have been considered, in which the permanent electric direction (of the given Lorentz force) is periodically overcompensated by the $\mathbf{E}$-field of an equally oscillating surface-charge layer. Observationally, the existence of pulsar winds has been proven via the (frequent) presence of bowshocks, and indirectly via the fact that all isolated pulsars spin down. To me, the ultimate solution of the wind-formation problem (of pulsars, offered in [Kundt, 2014c, 2016]) involves the unavoidable, permanent surface bombardment by downward electric currents - of 
periodically alternating sign - which steadily creates a marginally relativistic pair corona (of scale height $\mathrm{H}=10^{4} \mathrm{~cm} \mathrm{~T}_{7}$ ), escaping from it at trans-relativistic speeds. Such a trans-relativistically escaping pair corona forms a steady, almost neutral, almost axi-symmetric pair-plasma wind.

This wind implies a pulsar's broadband emission pattern, from radio waves up in frequency towards $\gamma$-ray energies, whereby its angular dependence is controlled by the geometry of its magnetosphere. Is this geometry dipolar? Most likely not, because its surface field is most likely stabilised by a toroidal magnetic bandage, and because the (broadband) emission-pattern changes from pulsar to pulsar: No uniform magnetospheric geometry (of pulsars) is indicated. Perhaps our Sun is a better example, with its oscillating multipolar magnetic surface structure, on the timescale of 22,2 years. PSR winds form another top invention of the inorganic Universe.

(9) Recycling of pulsars: is an often mentioned mechanism to form fast-spinning pulsars, of spinperiods in the msec range, because there has been an epoch when theorists were not sure how to create such extremely energetic dwarf stars: solar-mass objects spinning with trans-relativistic kinetic energies. But is such an extra mechanism really required? How do all their less rapid siblings receive their spins at birth, and do not all of them gradually spin down, even when occasionally, matter is transferred rapidly to them from a binary companion? In my preferred SN model, neutron stars are formed by the sudden gravitational collapse of the rapidly spinning, white-dwarf-like degenerate core of a sufficiently massive star, squeezed by the weight of its overlying stellar envelope [Kundt, 1998a, 2008]. This model predicts neutron-star spin periods $\mathrm{P}$ at birth all the way down to $\mathrm{P} \gtrsim 1 \mathrm{msec}$ - consistent with the data - from the constraint that there remain enough kinetic energy to eject the envelope of its progenitor star. And moreover, independent explanations (of achieving spinup towards $1 \mathrm{msec}$ ) - via mass accretion (from a companion star) - fail completely to achieve this goal: None of the dozens of well-observed neutron-star binaries in our Galaxy has ever been observed to spin up continuously throughout epochs longer than some ten years; they all oscillate in spin frequency, around (present-day) equilibrium spin periods, enforced by simultaneously acting braking torques. "Recycling" of pulsars looks to me like an unrealistic expectation, perhaps even conflicting with the Second Law.

(10) FRBs: standing for "Fast Radio Bursts", are rare, loud radio signals of very short (msec) duration, in the $\mathrm{GHz}$ range, of super-Galactic dispersion measure, preferentially arriving from higher Galactic latitudes, known since the year 2007, of which no more than 17 are presently (1 July 2016) known. Who emits them? From how far do they come?

Standard knowledge interprets the dispersion measure as a measure of distance, so that the FRBs would reach us from cosmologically far. But then, why have we never detected a similar signal from somewhere nearby, of gigantic brightness ?!? For this - cosmic homogeneity - reason, I prefer to re-interpret the dispersion measure as a measure of extreme coherence - in rare cases like this one - and wonder if not the CRs of ultrahigh energy $\left(\gtrsim 10^{20} \mathrm{eV}\right)$ could be the culprits, in regions of comparatively high density - like the upper atmosphere of Earth - where they can kick off a huge number of outer electrons from terrestrial molecules, at transrelativistic speeds, in their forward (tangential) direction. Why would such rare super events avoid infall directions near the Galactic plane? Perhaps because otherwise, the impacting UHE CRs would already have been 'discharged' before they reach our solar system, on their way through the (denser ISM medium in 
the) Galactic disk. Note that here, we are dealing with a quite rare phenomenon, whose explanation may involve rare circumstances.

(11) Gravitational-Wave Signals, like GW 150914, were first recorded at Earth on 14 September 2015, of duration 1/4 second, (and a second time on 26 December of that same year). Where did they come from? The (present) literature speaks of massive stellar-mass BHs (:= Black Holes), at distances of order Gpc, in neglect of certain literature since 2009, like Scientific American, which denies the possibility of their formation, [Kundt, 2015a]. Instead, it is my claim since March 2016 that we deal with the long-expected coalescence of (mostly $\lesssim 30 \mathrm{Mpc}$ distant) neutron-star binaries. With this problem, we deal with perhaps the most difficult theoretical-physics situation ever, a highly non-trivial calculation within Einstein's second-order GRT, which has so far only been handled by our best computers. In addition, at high order in the point-mass approximation for two compact masses on circular orbits around each other, the "chirp mass formula" has been derived for quantitative estimates (by Thibault Damour). It expresses the time derivative of the orbital frequency as an analytic function of the other determining quantities, and has stood its test for a few well-studied binaries, but loses its applicability during the fusion event, when tidal forces destroy the pointlike assumption for the two orbiters. Instead, the mere Kepler problem still applies at the onset of the fusion of two neutron stars, at their separation of $\lesssim 10^{2} \mathrm{~km}$, and nicely conforms with the measurements; as did the 2 BHs-description in the mainstream literature; [Kundt \& Marggraf, 2014]. But convincing interpretations must not content themselves with only a satisfaction of sufficient constraints, in particular when a less phantomic alternative interpretation has been in the literature for decades.

\section{Geophysical alternatives}

(12) The Tunguska catastrophe of 30 June 1908: what happened? At around 7 o'clock in the morning of 30 June 1908, hell broke loose in the Siberian taiga in an area of the size of Germany's Saarland. It roared and blew, fires were lit, and huge numbers of trees were damaged or felled, though selectively so in different locations (of the mountainous region). Only few people were hurt, but some cattle burnt, and several people and horses were knocked to the ground or onto their knees, out to distances of $\gtrsim 65 \mathrm{~km}$. Only decades after the event - and after a large number of (summerly) expeditions to the area, (initially led by Leonid Kulik), as well as from studies of eyewitness reports, and of scientific measurements at earthquake stations - did it become clear to insiders (like Andrei Ol'khovatov) that the holocaust had not struck from above, via the impact of a comet, or asteroid, but rather from below, by a volcanic outburst, a kimberlite, a more than 20-times more frequent event than infalls at the same destruction energy. Among the more than 30 reasons against an impact are 1) the almost radial treefall pattern, following the ridges and valleys, of net momentum zero, with 2) its $\geq 5$ centers, 3) its 'amphi-theatre', 'cauldron', and 'Merrill circus' geometry, its 4) 'telegraph posts'(formed by supersonic gusts, like at Hiroshima), with islands of survival, and 5) its characteristic 'Krinov profiles' along the slopes whereby the tree destruction was nearly complete at their tops, and declined to nearly zero destruction in the valleys. Further criteria were 6) the reports that the sounds ( $\gtrsim 20$ 'Barisal guns') of the holocaust preceded the 'columns of fire' in the sky, (unlike if caused by an infall), that 7) they lasted for more than $1 / 4$ 
hour (rather than for a few seconds), that 8) a strong shock wave raced all around the globe at soundspeed, that 9) instead of any impact crater, many conical lakes were found (like Suslov's hole), and many root stumps, plus a (10 ton) heavy (John's) rock which had been hurled through large distances from the cauldron, but 10) less than $10^{-10}$ (in mass) of extraterrestrial matter has ever been found. 11) There were the 3.5 bright nights in Europe and western Asia, illuminated by scattered sunlight from near the ionosphere, (caused by snowlike condensations from natural gas), as was likewise reported during the 1883 eruption of Krakatao's volcano. And 12) there are the several present-day recognitions - partially found by observations from space - that the site near the Stoney Tunguska (Kulikovskii crater) forms a center of various activities of the Asian continental plate, (such as the asiatic heat current, geomagnetic anomalies, MOHO isohypses, and intersection of fold lines), with repeated volcanic outbursts in the past; [Kundt, 2001, 2007b]. The site of the destructions was predetermined by our home planet.

(13) Plate Tectonics: Why is our friendly home planet Earth occasionally shaken by serious earthquakes, haunted by ejections of red-hot lava, or drastically ruptured by tsunamis - as happened last on 11 March 2011, near Japan's nuclear-power station Fukushima. Such terrestrial catastrophes have already caused enormous damage to civilised life, because they had not been foreseen in time for evacuations, but on the other hand, our biosphere would not have come into existence without the stirring action of volcanism which has equipped the surface of Earth with the $\gtrsim 40$ different chemical elements necessary for life: for plants, animals, and for people. Why is this so? What propels plate tectonics?

Geophysicists of the 19th century - like Alfred Wegener, and Harold Jeffreys - noticed that plates move, but did not understand how they could do so: None of the forces experienced near the surface of Earth are strong enough to make the continental plates move, at speeds of $\lesssim$ several $\mathrm{cm} / \mathrm{yr}$; they fail by many orders of magnitude. In order to make a stripe of oceanic crust move, say, of height $80 \mathrm{~km}$, and of comparable width, parallel to the observed system of transform faults, you need forces comparable in strength to those acting at depths of the liquid core, some $3000 \mathrm{~km}$ below NN. The global pattern of mid-ocean spreading lines (under high mountain 'ridges'), traversed by transform faults, with its corresponding system of subduction zones, suggests that plates move stepwise away from the spreading lines, one meter per century on average, forced by instantaneous gigantic forces at successive points of the spreading lines; see figure 5. Simple calculations show that such gigantic forces result - for a few seconds per century only - when hot fluid rises from the molten core of Earth, melting its way up from the thermally unstable core-mantle boundary, and reinforced by weight-reducing methane inclusions such that the weight of the growing tubes is much smaller than that of their surrounding, cool, solid mantle rocks. These growing hot, light, liquid vertical tubes exert enormous, growing overpressures on their surrounding rocks - quite similar to the feeding tubes of the $\sim 12$ volcanic island arcs of the Pacific, of which the Hawaiian one is the best known, except that the arcs are (more) discrete - and recur quasi-periodically along the system of spreading lines, roughly once every 100 years. A simple Newtonian calculation arrives at the equation of free fall, and shows that forced by the growing gigantic pressure near the top of a rising tube, its two opposing stripes of the crust can be instantly pushed apart to both sides through distances comparable with the tube's height, by typically 0.5 meter to either side, within one second; i.e. through horizontal distances of order $2000 \mathrm{~km}$ (!). Beyond such distances, further 


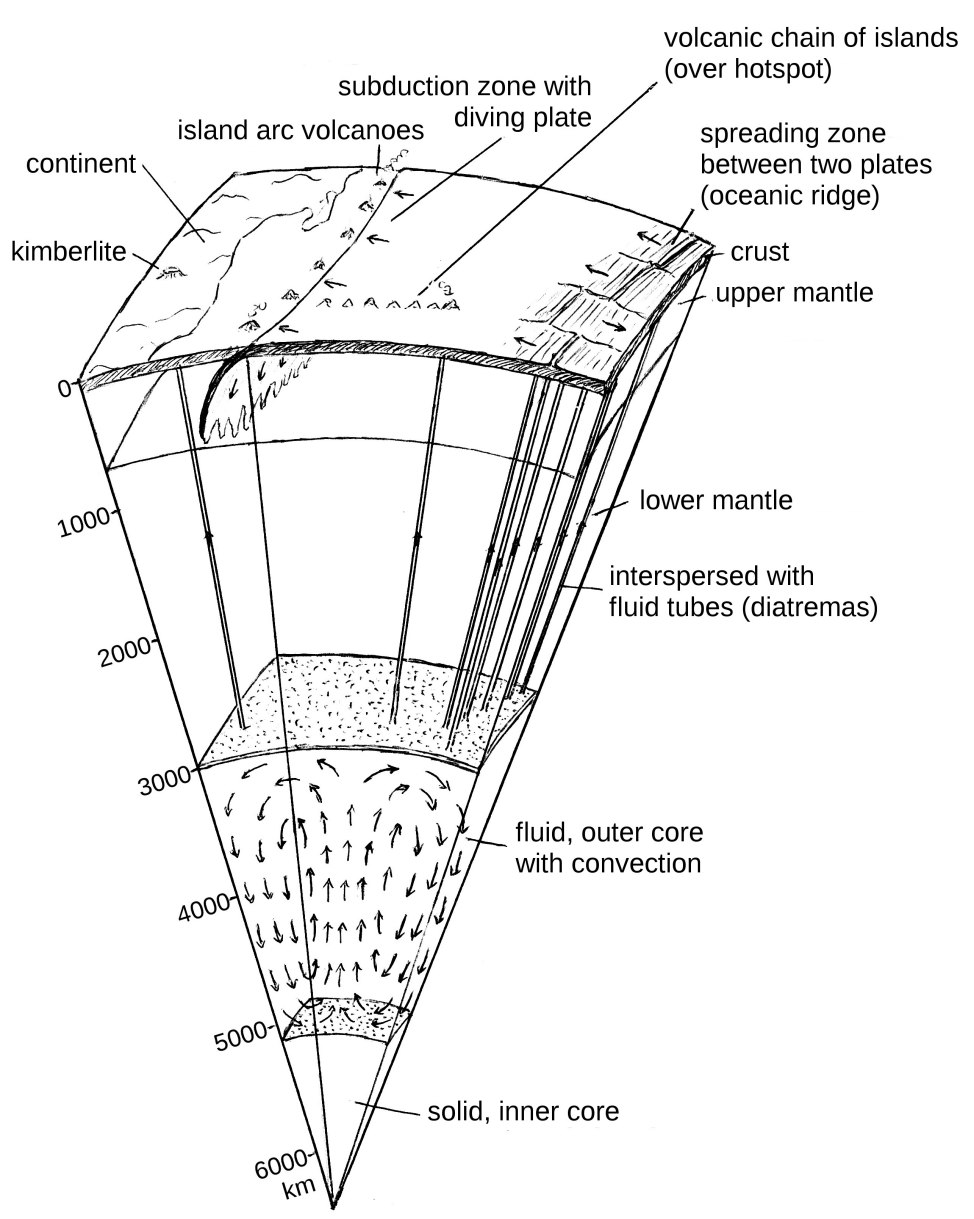

Figure 5: Expected geometry of the Earth's interior, giving rise to plate tectonics, and island-arc volcanoes, powered by hot vertical tubes (diatremes) which force their way up from the (unstable) core-mantle discontinuity. Reproduced from Klaus Strobach [1991], and [Kundt, 1998c, 2007a].

tensions tend to stay frozen into the crust for years to come, ready to relax via future earth quakes. These distances and times (of $0.5 \mathrm{~m}$ in one sec) of a quake are plausible because larger speeds (of pushing) would require too much kinetic energy - scaling as velocity squared - whereas weaker pressures would not make the rock move; [Kundt \& Jessner, 1986], [Kundt, 1998c, 2007a]. It is not all that easy to make the plates move.

\section{A biophysical alternative}

(14) The Hearts of the Plants: do such exist? I claim they do, and so did the Indian Acharya Jagadish Chandra Bose, already at around 1918. The hearts allow a plant to pump its dissolved nutrients all the way up from the soil, in which it is rooted, to its crown, typically some ton of water per day for tall trees, through all its xylem tubes, in order to feed and water its leaves, blossoms, fruits, branches, stems, and roots. They do so via osmotic tensions, up to heights of $\lesssim 140 \mathrm{~m}$ (for eucalyptus and redwood trees), corresponding to (required, and measured) root pressures of $\lesssim 14$ 

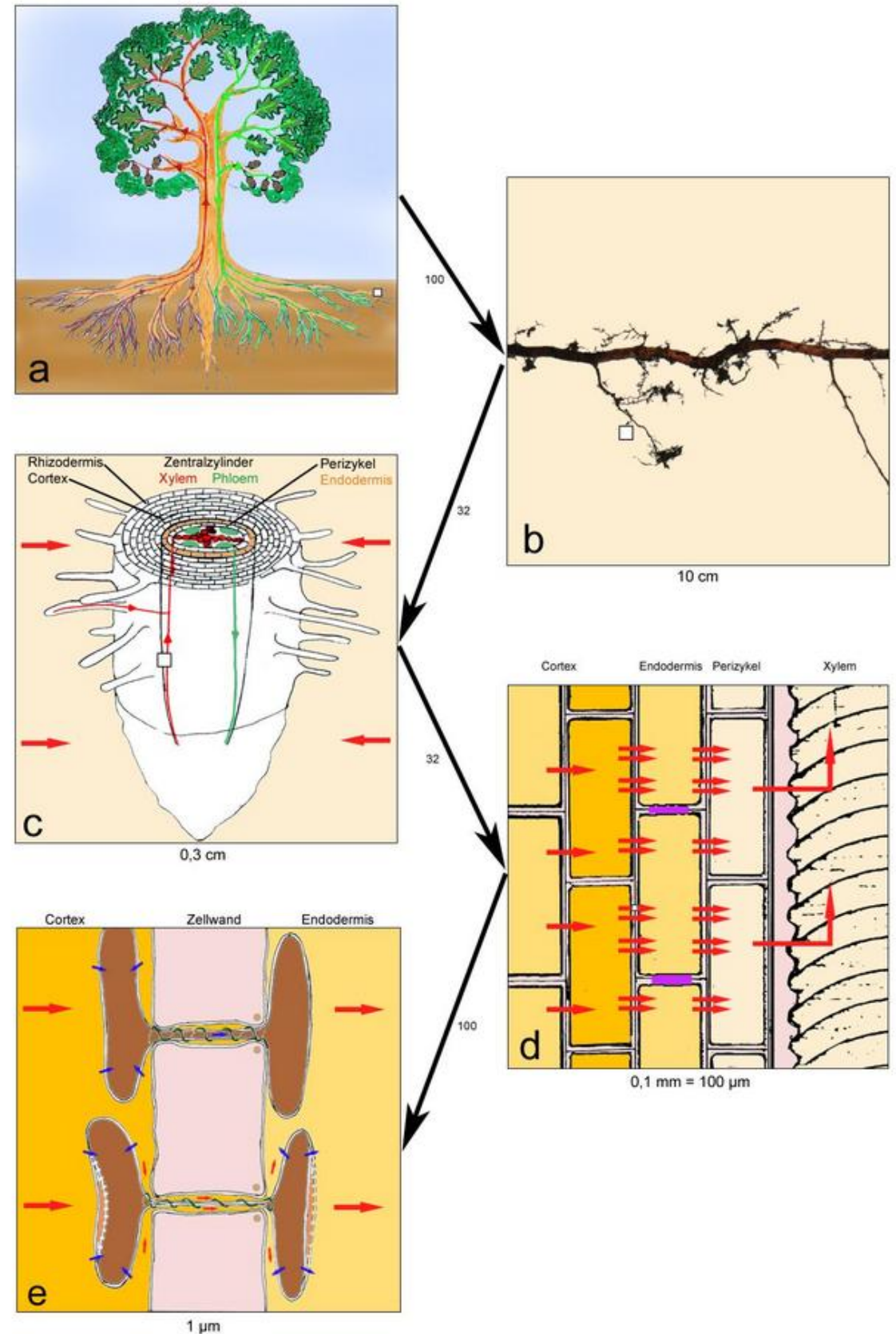

Figure 6: Water circulation in Plants, or rather tall Trees, powered by osmotic tensions, and propelled by (billions of) submicroscopic pumps in their endodermis, their 'hearts'. Taken from [Kundt \& Marggraf, 2014].

bar, whereby most of the weight of the rising water column is transferred to the reinforced walls of its xylem tubes at regular spatial intervals, via safety valves, and inclined tracheids, (or bordered pits, or perforation plates), and whereby a huge number of plasmodesmata, or vacuoles, (or pores, the 'hearts'), performs a reverse osmosis in the endodermis - or even additionally in the exodermis 
- of the roothair zones, at the tips of all the roots, which power the permanent sucking intake of ground water, and its subsequent osmotic lifting towards their crown.

This insight is not at all commonplace in the literature. Instead, mainstream biology was impressed by flowers in vases which emptied their water-filled containers rather quickly, seemingly via 'transpiration', (in reality osmotically, through their stalks). Biologists did not realise that transpiration is a diffusive process in the gas phase, whose efficiency cannot compete with the (ordered) osmotic transport in the liquid phase, at $10^{3}$ times higher mass densities; and that the moisture of leaves in the crowns of trees may have reached the crown via guttation - via the ordered shedding of excess pure water squeezed out at their upper ends from the xylem vessels through specific valves - rather than by sweating.

So billions, or even trillions of microscopic water pumps (in the endodermis of the outer root system of a big plant) can absorb ground water osmotically into its root ends - performing reverse osmosises - and push this water ahead through its xylem vessels, up into all its overground organs, through overall distances of between centimeters and hundreds of meters. It's a permanent large-scale flowsystem, to be described as a downhill flow according to the water potential $\Psi:=\rho \mathrm{g} \mathrm{z}+\mathrm{p}-\pi-\tau$, whose driving term is the osmotic pressure $\pi ; \rho:=$ mass density, $\mathrm{g}:=$ gravity acceleration, $\mathrm{z}:=$ height, $\mathrm{p}:=$ pressure, and $\tau:=$ capillary suction. Soil water tends to be attracted by a $\pi$ of order 7 bar (for typical plants) at the outer root ends, against its equilibrium value in the ground, and is subsequently pulled up vertically by again rising values of $\pi$ up to $\lesssim 14$ bar; see fig. 6. This circulation of (nourishing) water in plants parallels in many ways the circulation of (nourishing) blood in animals; it looks like a necessary condition for life, [Kundt, 1998b], [Kundt \& Marggraf, 2014]. Organisms require hearts to function.

\section{Conclusions}

Modern ( $~ 20$ th century) physics has increasingly entered an era of fast conclusions, in which progress is so fast, and specialisations are so manyfold, that healthy scepticism, careful control, and unbiased refereeing do no longer prevent wrong conclusions from entering into our foremost scientific literature. This talk (at Mondello) sketches 14 such 'alternative interpretations', or 'fashions', mainly from astrophysics, but even from geophysics, and biophysics, which should serve as a warning for any serious frontline scientist.

Acknowledgements: My sincere thanks go to Ole Marggraf, Hans Baumann, and Udo Wernick.

\section{References}

[1] Horn, Susanne, Kundt, W.: Magnetically Tilted Accretion Disks, Astrophysics \& Space Science 158, 205-221, 1989.

[2] Kundt, W.: Jets from Stars and Burning Disks, Lecture Notes in Physics 471, Springer, 1996.

[3] Kundt, W.: Astrophysics of Neutron Stars: Facts \& Fiction about their Formation and Functioning, Fundamentals of Cosmic Physics 20, No.1, pp.1-119, 1998a.

[4] Kundt, W.: The Hearts of the Plants, Current Science 75, pp.98-102, 1998 b. 
[5] Kundt, W.: Understanding Physics, Copernicus Gesellschaft, ed. Arne K. Richter, 1998c.

[6] Kundt, W.: The 1908 Tunguska Catastrophe: an alternative explanation, Current Science 81, pp.399-407, 2001.

[7] Kundt, W.: Jordans “Ausflug” in die Geophysik, in: Pascual Jordan (1902-1980), Mainzer Symposium, pp.123-131, 2007a.

[8] Kundt, W.: Tunguska (1908) and its Relevance for Comet/Asteroid Impact Statistics, in: Comet/Asteroid Impacts and Human Society, Springer, pp.331-339, 2007b.

[9] Kundt, W.: Supernovae, their functioning, lightcurves \& remnants, in: New Astron. Rev. Vol. 52, Issues 7-10, pp.364-369, 2008.

[10] Kundt, W.: The Sources of the Cosmic Rays, \& of the Gamma-Ray Bursts, after more than 440, 30)years of deliberation, in: Conference Proceedings Vol. 98, SIF, Bologna, pp.363-382, 2009.

[11] Kundt, W.: The local-Galactic interpretation of the GRBs, Memorie S.A.It. 81, N.1, pp.449-459, 2010 .

[12] Kundt, W.: ISM, Cosmic Rays, \& the Shape of the Heliosphere, MEMORIE S.A.It.83, pp.38-44, 2012.

[13] Kundt, W.: Astrophysics without Black Holes, and without Extragalactic GRBs, Acta Polytechnica $1,(1)$, pp. 27-33, 2014a.

[14] Kundt, W.: The Inorganic Machines of the Universe, XVIIth Brazilian School of Cosmology, Cambridge Scientific Publishers, pp.193-202, 2014b.

[15] Kundt, W.: How do Pulsars blow their Winds?, Bonn Workshop, 27.10.2014, 2014c.

[16] Kundt, W.: A brief Observational History of the Black-Hole Spacetimes, Advances in Mathematical Physics, Article ID 617128, 2015a.

[17] Kundt, W.: A Uniform Description of All the Astrophysical Jets, Mondello 2014, PoS(FRAPWS 2014) $025,1-9,2015 b$.

[18] Kundt, W.: How do Pulsars blow their Winds?, Mondello workshop, May 2015, PoS(MULTIF 2015) 006, 1-7, 2016.

[19] Kundt, W., Jessner, Axel: Volcanoes, fountains, earth quakes, and continental motion - what causes them?, Journal of Geophysics 60, 33-40, 1986.

[20] Kundt, W., Krishna, Gopal: The Physics of ExB-Drifting Jets, J. Astrophys. Astron. 25, 115-127, 2004.

[21] Kundt, W., Marggraf, Ole: Physikalische Mythen auf dem Prüfstand, Springer, 2014.

[22] Kundt, W., Yar, Aylin: Fireworks in Orion, Astrophys. Space Science 254, 1-12, 1997.

[23] Strobach, Klaus: Unser Planet Erde, Gebrüder Borntraeger, 1991. 


\section{DISCUSSION}

Elisabete M. de Gouveia Dal Pino: With regard to jet formation, are you talking about jet formation out of magnetic field lines arising from an accretion disk? But this is essentially the Blandford-Payne mechanism, isn't that so?

Kundt, Wolfgang: No, by no means! Even though Mitchell Begelman, Roger Blandford, and Martin Rees have not referred to my publications in their list of 813 references on the "jet/massive black hole" approach to the "theory of extragalactic radio sources" in Rev. Modern Physics Vol.56, No.2, part 1, April 1984, pp. 255-351, my understanding of the jet phenomenon works quite differently: with massive, magnetised rotators, in the distinct absence of black holes. Magnetic reconnections are required for creating continually relativistic pair plasma, and self-generated, toroidal magnetic fields are required for guiding its long-distance $\mathbf{E} \times \mathbf{B}$-drift through its (self-rammed) channels. But these, and all the additional processes inside a jet engine are believed to occur spontaneously, without any preset facilities. 